Research Article

\title{
Free Radical Scavenging Capacities and Alleviating Actions of Polysaccharides Extract of Termitomyces le-testui on Methylprednisolone-Induced Immunodepression in Rats
}

\author{
Foncham Evans Ngwenah, ${ }^{1}$ Kada Sanda Antoine, ${ }^{2}$ Salah Martin, ${ }^{2}$ \\ Tume Christopher, ${ }^{1}$ and Oumar Mahamat $\mathbb{D}^{2}$ \\ ${ }^{1}$ Laboratory of Biochemistry, Department of Biochemistry, Faculty of Science, University of Bamenda, Bamenda, Cameroon \\ ${ }^{2}$ Laboratory of Biological Sciences, Department of Biological Sciences, Faculty of Science, University of Bamenda, \\ Bamenda, Cameroon
}

Correspondence should be addressed to Oumar Mahamat; oumahamat@yahoo.com

Received 12 July 2021; Accepted 19 October 2021; Published 8 November 2021

Academic Editor: Kuldeep Singh

Copyright (c) 2021 Foncham Evans Ngwenah et al. This is an open access article distributed under the Creative Commons Attribution License, which permits unrestricted use, distribution, and reproduction in any medium, provided the original work is properly cited.

\begin{abstract}
Background. Natural products have been said to show immunomodulatory and antioxidant activities. The research study was aimed to assess the immunomodulatory and free radical scavenging activities of crude polysaccharide from dry mushroom fruiting bodies of Termitomyces le-testui. Materials and Methods. Hot water extract of polysaccharide extract of T. le-testui was prepared and tested in white albino Wister rats for its immunomodulatory activities effect on methylprednisolone-immunosuppressed animals. In addition, the radical scavenging activity of the polysaccharide was evaluated using nitrite and hydrogen peroxide. Results. The result of the study showed that the polysaccharide T. le-testui increases the phagocytic index, energy metabolism of macrophages, spleen index, and nitric oxide in a concentration-dependent manner in immunosuppressed animals. Also, it was observed that the extract increased dose-dependent total oxidative stress and thymus index. Finally, the crude polysaccharide-rich extract showed nitrite and hydrogen peroxide scavenging activity in a concentration-dependent manner. Conclusion. Polysaccharide-rich extract possesses immunomodulatory and antioxidant properties.
\end{abstract}

\section{Introduction}

Natural products, especially plants, are one of the immunomodulators that can provide an alternative to conventional chemotherapy for a large variety of diseases. As immunomodulators, they are especially important when the host defense mechanism has to be stimulated in cases of the impaired immune response. Some of them are important when a selective immunosuppression is desired. Mushrooms have been utilized in folk medicine since ancient times [1], but this concept gained little more credibility in the last decades as they exhibit significant therapeutic effect including antioxidant and immunomodulatory activities. This is partly due to a large number of substances with potential immunomodulatory activities that mushrooms possess $[2,3]$.

Mushrooms, as immunomodulators, they can suppress or enhance the immune system activities [4], and this offers them a large use to treat infections [5]. Among derived mushroom substances, there is ample evidence that polysaccharides exhibit various bioactivities such as antitumor, anticancer, antiviral, antibacterial, antifungal, anticoagulant, and immunological activities $[6,7]$. Lentinan from $L$. edodes and G. lucidum polysaccharide, for example, through their immunostimulatory activities, showed important roles in maintaining tissue homeostasis and fighting diseases $[8,9]$. In addition to the immunomodulation, polysaccharides also play an important role in the control of oxidative stress 
[10-12], thus contributing to the improvement of the immune response.

T. le-testui is an edible mushroom commonly consumed in Cameroon. The dry matter of $T$. le-testui revealed that it is made up of $59.02 \%$ of total carbohydrates, $23.6 \%$ of total sugars, and $0.84 \%$ of reducing sugars [13]. Data from various studies reported the immunomodulatory activities of $T$. le-testui $[3,14]$. However, there is no data available on the active constituents of T. le-testui. Polysaccharides may be the bioactive constituents, but this remains to be demonstrated. The present study was therefore undertaken to assess the immunemodulatory activities against methylprednisolone-induced immunosuppression in rats and in vitro antiradical activities of polysaccharides extract of $T$. le-testui.

\section{Materials and Methods}

\subsection{Materials}

2.1.1. Mushroom Specimen. Fresh head bodies of Termitomyces le-testui (Figure 1) were obtained in the local market of Mbouda, in the West Region of Cameroon. Mushroom samples were authenticated by Dr. Njuonkou Andre Ledoux, a botanist in the Department of Biological Sciences, Faculty of Sciences, of the University of Bamenda, Cameroon. The obtained mushrooms were washed with tap water and cut into small pieces.

2.1.2. Preparation Polysaccharides. Polysaccharides were extracted using hot water [15]. Fresh mushrooms were fully immersed in distilled water and incubated at $95^{\circ} \mathrm{C}$ in a water bath for six hours. The preparation was filtered and the filtrate was used to obtain the polysaccharides extract. The filtrate was mixed with ethanol (70\%) at equal volumes and kept overnight at $4^{\circ} \mathrm{C}$ to precipitate the polysaccharides. After precipitation, the mixtures were then centrifuged at $1800 \mathrm{rpm}$ during $10 \mathrm{~min}$ at $4^{\circ} \mathrm{C}$ temperature, and the pellets were then collected and dried at $30^{\circ} \mathrm{C}$. The dried products representing the polysaccharide extracts were kept at about $4^{\circ} \mathrm{C}$.

2.1.3. Determination of Total Carbohydrate Content. The carbohydrate contents were determined by the slightly modified phenol-sulfuric acid method according to Masuko et al. [16]. Fifty microliters of crude polysaccharide solution was mixed with $150 \mu \mathrm{L}$ of concentrated sulfuric acid and immediately with $30 \mu \mathrm{L}$ of $5 \%$ phenol and then the mixture was kept at $90^{\circ} \mathrm{C}$ for $5 \mathrm{~min}$. The absorbance of the mixture after cooling to room temperature was measured at $490 \mathrm{~nm}$. The total carbohydrate content was calculated using a standard curve of D-glucose and it was found to be $75 \%$ of the dried extract.

\subsection{In Vitro Evaluation of Antioxidant Activities}

2.2.1. Scavenging Activity Determination. The antioxidant activities of polysaccharides extract of $T$. le-testui were analyzed by assessing the free radical scavenging activities.

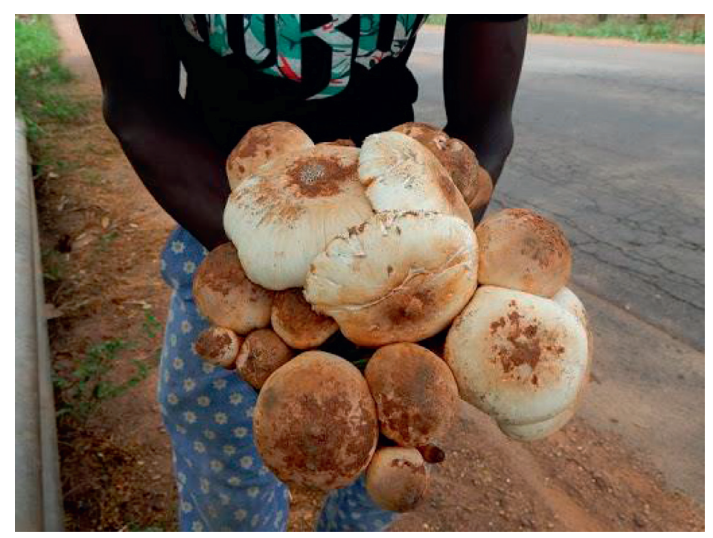

Figure 1: Photography of T. le-testui.

The absorbance values measured in a UV-Vis spectrophotometer were used to determine the percentage inhibition of free radical scavenging activity by using the standard formula $\left(\mathrm{F}_{1}\right)$ as reported by Bahatti et al. [17]:

$$
\left(F_{1}\right) \cdot \% \text { inhibition }=\left[\frac{A_{518}(\text { control })-A_{518}(\text { sample })}{A_{518}(\text { control })}\right] \times 100 \text {, }
$$

where $A_{518}$ (control) is the absorbance of control and $A_{518}$ (sample) is the absorbance of free radical + sample extract/standard.

2.2.2. Nitrite-Scavenging Activity. Nitrite-scavenging activity was evaluated based on the absorbance at $520 \mathrm{~nm}$ using a UV-spectrophotometer according to the method reported by Kato et al. [18]. One milliliter of $1 \mathrm{mmol} / \mathrm{L}$ $\mathrm{NaNO}_{2}$ (Sigma Co.) solution was added to $1 \mathrm{~mL}$ of each sample, and the resulting mixtures were adjusted to a $\mathrm{pH}$ of 2.5 using $0.1 \mathrm{~N} \mathrm{HCl}$ and $0.2 \mathrm{~N}$ citric acid solutions. Each sample was allowed to react at $37^{\circ} \mathrm{C}$ for 1 hour, after which $1 \mathrm{~mL}$ of each sample was taken from the solution and mixed thoroughly with $3 \mathrm{~mL}$ of $2 \%$ acetic acid and $0.4 \mathrm{~mL}$ of the Griess reagent. The solutions were stored at room temperature for $15 \mathrm{~min}$. The Griess reagent was prepared by mixing an equal amount of $1 \%$ sulfanilic acid (Sigma Co.) and $1 \%$ naphthylamine (Sigma Co.), which were made with $3 \%$ acetic acid.

2.2.3. Hydrogen Peroxide Scavenging Activity. The ability of the mushroom extracts to scavenge hydrogen peroxide was assessed according to Ruch et al. [19]. Mushroom extracts were added to $0.6 \mathrm{~mL} \mathrm{H}_{2} \mathrm{O}_{2}$ solution $\left(40 \mathrm{mM} \mathrm{H}_{2} \mathrm{O}_{2}\right.$ was prepared with phosphate buffer, $0.1 \mathrm{M}, \mathrm{pH}$ 7.4) and the total volume was made up to $3 \mathrm{~mL}$ and mixed properly. Then the absorbance was measured at $230 \mathrm{~nm}$ using a spectrophotometer. Phosphate buffer, without $\mathrm{H}_{2} \mathrm{O}_{2}$, was considered as blank solution.

2.2.4. Assay of Inhibition of Free Radicals Using Thiobarbituric Acid (TBA) Method. The TBA method was used to represent the inhibition of the production of carbonyl 
compounds degraded from the peroxides at a later stage. TBA test is used to measure the second product of peroxide oxidation such as aldehyde and ketone. The assay was done according to Kikuzaki and Nakatani [20]. To $2.0 \mathrm{~mL}$ of the sample solution, $1.0 \mathrm{~mL}$ of $20 \%$ aqueous trichloroacetic acid (TCA) and $2.0 \mathrm{~mL}$ of aqueous thiobarbituric acid (TBA) solution were added. The mixture was placed in a boiling water bath for $10 \mathrm{~min}$. After cooling, it was centrifuged at $3000 \mathrm{rpm}$ for $20 \mathrm{~min}$ and the absorbance was measured at $532 \mathrm{~nm}$ and recorded.

\subsection{In Vivo Immunological Tests}

2.3.1. Animals. Albino rats (Wistar strain) aging 7 to 8 months and weighing 100-250 g were obtained from the laboratory of physiology and pharmacology of the University of Dschang. The animals were housed in cages under controlled conditions of temperature and alternating 12 hours cycle of light and darkness. They were given free access to standard rat pellet food and tap water ad libitum. All animals were acclimatized to the working environment 1 week before the beginning of the experiment. The experimental protocol was approved by the Ethics Committee of the Department of Biological Sciences of the University of Bamenda.

2.3.2. Grouping and Treatment of Animals. After 1 week of acclimatization, rats were randomly distributed into 6 groups of 5 rats each. A group of 5 rats did not receive the immunosuppressive drug (methylprednisolone) (Group 1 or normal control). The remaining 5 groups of rats were all immunosuppressed by the injection of methylprednisolone via the tail vein $(1 \mathrm{~mL} / \mathrm{kg}$ of animal weight) before receiving the other treatments. One group of the immunosuppressed rats (Group 2 or negative control) received distilled water. Another group (Group 3 or positive control) received intraperitoneal injection of $\mathrm{BCG}\left(2.10^{3} \mathrm{CFU} / \mathrm{mL}\right)$ for the entire treatment period. Finally, the remaining immunosuppressed groups of rats (Groups 4, 5, and 6) were orally treated by gavage with the different doses of the mushroom extract 25 , 50 , and $100 \mathrm{mg} / \mathrm{kg}$, respectively. Treatment was done every day for 10 days.

2.3.3. Sample Collection. For this part of the study, two animal groupings were done. The first grouping was to evaluate the phagocytic index and lymphoid organ index, and the second grouping was to evaluate the energy metabolism in intraperitoneal macrophages, production of nitric oxide, and total oxygen radical. The treatment was done for 10 days. Thereafter, animals were administered a mixture of ketamine (dose) and Diazepam (dose) (ratio 1:3) and blood was collected by cardiac puncture in dry essay tubes to assess the serum nitric oxide and total oxygen radical or through the orbital venous to evaluate the phagocytic index. Peritoneal macrophages were obtained by washing the peritoneal cavity in the phosphate buffer. Spleen and thymus were collected and kept in formalin solution $10 \%$ for histological analysis.

2.3.4. Assessment of Phagocytic Index. Phagocytic index was assessed through the carbon clearance test as described by Honghui et al. [21] with some modification. Thirty rats treated as described previously were intravenously injected with diluted India ink at $100 \mu \mathrm{L} / 10 \mathrm{~g}$ body weight. Blood specimens were collected at $2 \mathrm{~min}\left(t_{1}\right)$ and $10 \mathrm{~min}\left(t_{2}\right)$ from the retinal venous plexuses and $20 \mu \mathrm{L}$ of the collected blood was then mixed with $2 \mathrm{~mL} 0.1 \% \mathrm{Na} 2 \mathrm{CO}$. The absorbance at $600 \mathrm{~nm}$ was measured on a UV-visible spectrophotometer with $0.1 \% \mathrm{Na} 2 \mathrm{CO} 3$ as the blank. The liver and the spleen were weighed, and the phagocytic index was calculated as follows:

$\left(F_{2}\right): K=\left(\lg \mathrm{OD}_{1}-\lg \mathrm{OD}_{2}\right) /\left(t_{2}-t_{1}\right)$, where $\mathrm{OD}_{1}$ was optical density for $t_{1}$ and $\mathrm{OD}_{2}$ was optical density for $t_{2}$ $\left(F_{3}\right)$ : phagocytic index $=\sqrt[3]{k} \times A /(B+C)$, where $\mathrm{A}$ is the body weight, $B$ the liver weight, and $C$ the spleen weight

2.3.5. Evaluation of Energy Metabolism in Peritoneal Macrophages. Peritoneal macrophages were harvested by washing the peritoneum in $3 \mathrm{~mL}$ of phosphate buffer saline (PBS). Peritoneal cells were immediately counted using a hemocytometer (Malassez cell). A cell suspension (density; $1 \times 10^{3}$ cells $/ \mathrm{mL}$ ) was prepared and $2 \mathrm{~mL}$ of this suspension was mixed with $1 \mathrm{~mL}$ of MTT (3-(4,5-dimethylthiazol-2-yl)2,5-diphenyl tetrazolium) solution (1\% in PBS). After 2 hours of incubation, $1 \mathrm{~mL}$ of $1 \mathrm{~N} \mathrm{HCl}$ was added and the mixture was allowed to stand for $15 \mathrm{~min}$ after which the optical density was read at $540 \mathrm{~nm}$. The mean of the optical density in treated animal was compared to that of control to evaluate the energy metabolism of the cells (reference).

2.3.6. Evaluation of Radical Compounds Production. The crude polysaccharides of $T$. le-testui were analyzed for their effect on radical compounds production. This test is based on iron catalyzed breakdown of hydroperoxides into alkoxyl (RO.) and peroxyl (ROO.) radicals which react with the chromogen ( $\mathrm{N}, \mathrm{N}$-dimethyl - $\mathrm{p}$ - phenylenediamine sulphate) towards formation of a colored compound, the absorbance of which is photometrically detectable. The intensity of the color correlates directly with the quantity of radical compounds, according to Lambert-Beer's law, and it can be related to the oxidative status of the sample. This was done by the method described by Pinaki et al. [22]. The method makes use of chromogen reagent solution $(23.5 \mathrm{mg}$ of N,Ndimethyl- p-phenylenediamine sulphate (Aldrich, Sigma) in $10 \mathrm{~mL}$ of 20 millimolar PBS ( $\mathrm{pH} 7.4)$ ) and kept in $4-8^{\circ} \mathrm{C}$. Briefly, 100 microliters of plasma diluted to 20 times in PBS was dissolved in $1 \mathrm{~mL}$ of acetate buffer. 25 microliters of working chromogen solution was added, and absorbance was taken at $505 \mathrm{~nm}$ by $6 \mathrm{~min}$ time-scan in UV-Vis spectrophotometer. The absorbance value was obtained for each 
sample against blank and compared to the curve obtained using $\mathrm{H}_{2} \mathrm{O}_{2}$.

2.3.7. Evaluation of Nitric Oxide Production. The crude polysaccharides of T. le-testui were analyzed for their effect on nitric oxide production as described by Pinaki et al. [22]. Griess reagent (made by mixing an equal amount of $1 \%$ sulfanilic acid and 1\% naphthylamine which was made with $3 \%$ acetic acid) is the principal reagent used. The collected serum sample was made up to $1 \mathrm{~mL}$ using $20 \%$ phosphate buffer. To each sample of serum, $1 \mathrm{~mL}$ of the working reagent (Griess reagent) was added and allowed to stand for $5 \mathrm{~min}$ and absorbance was measured at $540 \mathrm{~nm}$. This was done in duplicate for each sample, and the absorbance was recorded. A standard curve was produced using $\mathrm{NaNO}_{2}$ as standard solution.

2.3.8. Histological Analysis of Spleen and Thymus. Spleen and thymus of sacrificed animals were collected, washed in cold phosphate buffer saline, and blotted dried. They were weighted to obtain the absolute organ weight. The organ index was calculated as follows:

$$
\left(F_{4}\right) \cdot \text { organ index }\left(\frac{\mathrm{mg}}{\mathrm{g}}\right)=\frac{\text { (weight of thymus or spleen) }}{\text { body weight }} \text {. }
$$

Organs were immediately fixed in $10 \%$ formalin until the day of histological analysis. Later, the fixed tissues were dehydrated and then the samples were cleared in 2 changes of xylene. Samples were then impregnated and embedded in paraffin, then embedded, and blocked out. Sections of 4-6 $\mu \mathrm{m}$ thickness were cut using a rotary microtome and mounted on glass slides. After staining with Hematoxylin and Eosin $(\mathrm{H} \& \mathrm{E})$, the sections were imaged using the Pannoramic Viewer (3DHISTECH, Budapest, Hungary) connected to a microscope.

2.4. Statistical Analysis. Experimental data obtained were expressed as the mean \pm standard deviation of the experiment done in triplicate. Data was analyzed using one-way analysis of variance, followed by post-Students tests. Values of $p<0.05$ were considered as indicative of significance. All calculations were carried out using the GraphPad Prism ${ }^{\circledR}$ V5.03 software (GraphPad Software Inc. ${ }^{\circledR}$, CA, USA).

\section{Results}

3.1. Nitrite and Hydrogen Peroxide Scavenging Activity of Crude Polysaccharides of $T$. le-testui. The polysacchariderich extract of $T$ le-testui was tested for its antiradical activity by evaluating its effect on nitrite and hydrogen peroxide scavenging activity (Table 1 ). The result showed that a concentration-dependent increase of the inhibition percentage of free radical activity was observed with extract. This activity of polysaccharides was similar to the effect of vitamin C (100 mg) at $11.06 \mathrm{mg} / \mathrm{mL}$ polysaccharides extract
TABLE 1: Radical scavenging activity of crude polysaccharides of T. le-testui as measured by the nitrite and $\mathrm{H}_{2} \mathrm{O}_{2}$-scavenging tests and compared to standards (vitamin C).

\begin{tabular}{lcccc}
\hline \multicolumn{2}{c}{$\begin{array}{c}\text { Nitrite-scavenging } \\
\text { activity }\end{array}$} & \multicolumn{2}{c}{$\mathrm{H}_{2} \mathrm{O}_{2}$-scavenging activity } \\
& $\mathrm{OD}$ & $\%$ & $\mathrm{OD}$ & $\%$ \\
\hline Extract $(\mathrm{mg} / \mathrm{mL})$ & & & & \\
8.3 & $1.33 \pm 0.04$ & $9.05 \pm 3.27^{*}$ & $0.59 \pm 0.06$ & $12.07 \pm 0.58^{*}$ \\
$11.06 \mathrm{mg} / \mathrm{mL}$ & $0.76 \pm 0.05$ & $48.15 \pm 3.47$ & $0.54 \pm 0.001$ & $19.50 \pm 9.61$ \\
\hline Vitamin C $(\mathrm{mg} / \mathrm{mL})$ & & & \\
100 & $0.77 \pm 0.01$ & $47.26 \pm 1.14$ & $0.52 \pm 0.004$ & $21.16 \pm 0.96$ \\
\hline
\end{tabular}

Asterisks $\left({ }^{*} p<0.05\right)$ indicate the significant difference as compared to the positive control (vitamin C).

while $8.3 \mathrm{mg} / \mathrm{mL}$ was significantly different from the effect of vitamin $\mathrm{C}$.

\subsection{Free Radicals Scavenging Using Thiobarbituric Acid(TBA)} Method. The TBA method was used to evaluate the inhibition of the production of carbonyl compounds degraded from the peroxides at a later stage. The results showed that the polysaccharides active extracts of $T$. le-testui caused a decrease in the production of carbonyl compounds in a concentration-dependent manner (Figure 2). The production of carbonyl compounds was significantly less with the polysaccharides extract than the synthetic antioxidant vitamin C.

3.3. Effect of T. le-testui Crude Polysaccharide on Phagocytic Index and Peritoneal Cells Metabolism in MethylprednisoloneImmunosuppressed Rats. In vivo administration of methylprednisolone resulted in significant decreases $(p<0.05)$ in the phagocytic index and energy metabolism of peritoneal macrophages (Table 2). However, BCG as well as polysaccharides extract of $T$. le-testui treatments prevented the increase and maintained these parameters near the normal level and even above normal for the dose of extract of $100 \mathrm{mg} / \mathrm{kg}$. Polysaccharides extract treatments were also able to reverse the decreases in phagocytic index caused by methylprednisolone to near normal. No significant differences were observed with BCG treatment.

3.4. Effect of Crude Polysaccharide Extract of T. le-testui on Organ of Immune System. The treatment with methylprednisolone significantly decreased the spleen and thymus weight (Table 3). Administration of polysaccharides extract of $T$. le-testui significantly caused the increase of spleen index and thymus index at the doses of $100 \mathrm{mg} / \mathrm{kg}$ and $50 \mathrm{mg} / \mathrm{kg}$, respectively. The reversal effect of the extract brought back the organs to normal. There was no difference compared to the effect of BCG.

3.5. Serum Level of Nitric Oxide and Radical Compounds of Methylprednisolone-Immunosuppressed Rats Treated with the Crude Polysaccharide of $T$. le-testui. Administration of 


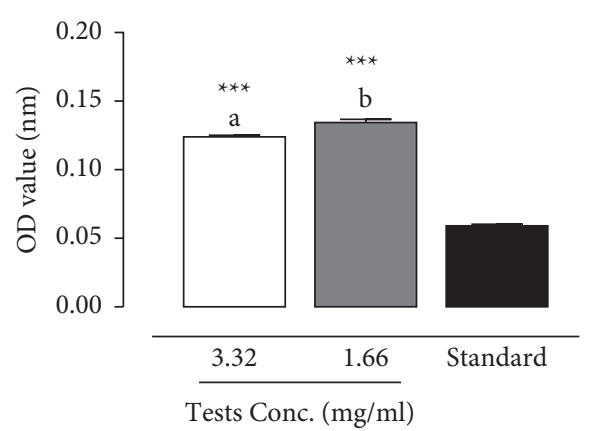

FIgURE 2: Antioxidant activity of polysaccharides extract of T. le-testui as measured by the TBA method at $532 \mathrm{~nm}$ and compared to standards (vitamin $\mathrm{C}$ ).

TABLE 2: Effect of T. le-testui crude polysaccharides on phagocytosis and energy metabolism in methylprednisolone-treated rats.

\begin{tabular}{lccc}
\hline Treatments & Dosage & Phagocytic index & $\begin{array}{c}\text { Energy } \\
\text { metabolism }\end{array}$ \\
\hline Nor. control & - & $12.56 \pm 0.64^{* *}$ & $0.11 \pm 0.028^{*}$ \\
Neg. control & $1 \mathrm{ml}$ & $5.31 \pm 0.49$ & $0.07 \pm 0.007$ \\
BCG $\left(2.10^{3} \mathrm{CFU} / \mathrm{mL}\right)$ & $200 \mu \mathrm{l}$ & $10.11 \pm 1.81^{*}$ & $0.11 \pm 0.014^{*}$ \\
& 25 & $13.84 \pm 5.82$ & $0.09 \pm 0.082$ \\
Extract $(\mathrm{mg} / \mathrm{kg})$ & 50 & $13.99 \pm 4.14^{*}$ & $0.21 \pm 0.071^{*}$ \\
& 100 & $18.73 \pm 4.73^{* *}$ & $0.15 \pm 0.079^{*}$ \\
\hline
\end{tabular}

Neg. control: prednisolone-treated animal and receiving the vehicle (saline solution) and Nor. control: normal control. Asterisks $\left({ }^{*} p<0.05,{ }^{* *} p<0.01\right.$, and $\left.{ }^{* * *} p<0.001\right)$ indicate the significant difference as compared to negative control.

TABle 3: The effect of crude polysaccharides extract of Termitomyces le-testui on spleen and thymus index.

\begin{tabular}{lccc}
\hline Treatment & & Spleen index & Thymus index \\
\hline Neg. control & $1 \mathrm{ml} \mathrm{H}_{2} \mathrm{O}$ & $0.0032 \pm 0.0008$ & $0.00019 \pm 0.00001$ \\
Nor. control & - & $0.0076 \pm 0.0005^{*}$ & $0.00041 \pm 0.00003^{*}$ \\
BCG & $200 \mu \mathrm{l}$ & $0.0043 \pm 0.0017^{*}$ & $0.00046 \pm 0.00005^{*}$ \\
$\left(2.10^{3} \mathrm{CFU} / \mathrm{ml}\right)$ & & & \\
& 25 & $0.0045 \pm 0.0005$ & $0.0010 \pm 0.00051$ \\
Extract & 50 & $0.0052 \pm 0.0001^{*}$ & $0.0010 \pm 0.00022^{*}$ \\
$(\mathrm{mg} / \mathrm{kg})$ & 100 & $0.0055 \pm 0.0005^{*}$ & $0.00081 \pm 0.0003^{*}$ \\
\hline
\end{tabular}

Neg. control: prednisolone-treated animal and receiving the vehicle (saline solution) and Nor. control: normal control. Asterisks $\left({ }^{*} p<0.05,{ }^{* *} p<0.01\right.$, and $\left.{ }^{* * *} p<0.001\right)$ indicate the significant difference as compared to negative control.

methylprednisolone to rats significantly decreased the serum $\mathrm{NO}$ and radical compounds level compared to normal animal. In concentration-dependent manner, treatment with polysaccharides extracts of $T$. le-testui resulted in significant increase in the levels of NO (Table 4). The effect of the treatment with polysaccharides extract was similar to that of BCG. Moreover, polysaccharides extract of T. le-testui reversed the effect of methylprednisolone with the highest effect observed with the lowest dosage.
3.6. Effects of Polysaccharides-Enriched Extracts of T. le-testui on Lymphoid Cells Accumulation in Spleen and Thymus Tissues. Methylprednisolone resulted in significant reduction in richness of lymphocytes in spleen and thymus of rats. In thymus, polysaccharides extract of $T$. le-testui treatment also increased the richness of lymphocytes (thymocytes) as compared to that of animals not treated (negative control). This was demonstrated by the dense thymus cortex observed in treated animals (Figure 3). The administration of polysaccharides extract of $T$. le-testui also affected the lymphoproliferative activity of spleen. The treatment has caused the white pulp to have a dense germinal center compared to the negative control (Figure 4).

\section{Discussion}

Since a long time ago, mushrooms have been known for numerous pharmacological activities. This also includes immunomodulatory and antioxidant activities [23]. In the present study, we assessed the reversal effect of crude polysaccharide extract on methylprednisolone-immunosuppressed effect in white albino Wistar rats and its antioxidant activities. Nowadays, there is considerable effort to identify natural substances that can protect against oxidative stress or stabilize or deactivate free radicals in an immunosuppressed state. Nitric oxide radical is one of the potent radicals that acts by generating full name of $\mathrm{NO}_{2}\left(\mathrm{NO}_{2}\right)$ [24]. The polysaccharide extract of $T$. le-testui was found to decrease the amount of radical. Moreover, the polysaccharides extract of T. le-testui has demonstrated a potential to have a $\mathrm{H}_{2} \mathrm{O}_{2}$-scavenging activity demonstrating that $T$. le-testui has potent antioxidant properties. Hydrogen peroxide $\left(\mathrm{H}_{2} \mathrm{O}_{2}\right)$, a biologically relevant, nonradical oxidizing species, can be formed in tissues through oxidative processes. $\mathrm{H}_{2} \mathrm{O}_{2}$ produced may result in the production of hydroxyl radicals $(\bullet \mathrm{OH})$ causing the lipid peroxidation [25] or alteration of the structure and function of many cellular components [26]. Thus, the polysaccharide-rich extract of T. le-testui may counteract the oxidative effect of $\mathrm{H}_{2} \mathrm{O}_{2}$. Further demonstration of the radical scavenging activity of T. le-testui was observed in TBA test assay. Though the scavenging activity of polysaccharide-rich extract of T. le-testui noticed in TBA assay test was lower compared to that of vitamin $C$, this result supports its radical scavenging potential. Together, the results of this study demonstrated the free radicals scavenging power of the polysaccharides extract of T. le-testui, as it has been reported for numerous natural products [27].

Several studies reported the immunomodulatory effects of the extract of Termitomyces species through a remarkable improvement of the antibodies production, and also the phagocytic activities $[3,14]$. In this study, the crude polysaccharides of $T$. le-testui were found to reverse the immunosuppressed effect of methylprednisolone. Firstly, this was demonstrated by increasing the phagocytic index towards the normal level in a dose-dependent manner. Secondly, the polysaccharides extract of T. le-testui may enhance the metabolism rate of peritoneal macrophages from the methylprednisolone-treated rats. Macrophages have a strong engulf particulate matter function in vivo or in 
TABLE 4: Effect of the crude polysaccharide of T. le-testui on serum level of nitric oxide and reactive oxygen species production in methylprednisolone-treated rats.

\begin{tabular}{lccr}
\hline Treatment & & NO & ROS \\
\hline Nor. control & - & $1.340 \pm 0.0028^{* * *}$ & $297.40 \pm 1.31^{* * *}$ \\
Neg. control & $1 \mathrm{ml} \mathrm{H} \mathrm{H}_{2} \mathrm{O}$ & $0.3151 \pm 0.062$ & $60.77 \pm 10.46$ \\
BCG $\left(2.10^{3} \mathrm{CFU} / \mathrm{ml}\right)$ & $200 \mu \mathrm{l}$ & $0.5557 \pm 0.0070^{*}$ & $125.7 \pm 18.88^{* * *}$ \\
& 25 & $0.7508 \pm 0.376$ & $235.2 \pm 41.19^{* * *}$ \\
Extract $(\mathrm{mg} / \mathrm{kg})$ & 50 & $1.016 \pm 0.524^{*}$ & $238.9 \pm 103.39^{* *}$ \\
& 100 & $1.045 \pm 0.343^{* *}$ & $218.7 \pm 69.20^{* *}$ \\
\hline
\end{tabular}

Neg. control: prednisolone-treated animal and receiving the vehicle (saline solution) and Nor. control: normal control. Asterisks $\left({ }^{*} p<0.05,{ }^{* *} p<0.01\right.$, and $\left.{ }^{* * *} p<0.001\right)$ indicate the significant difference as compared to negative control.

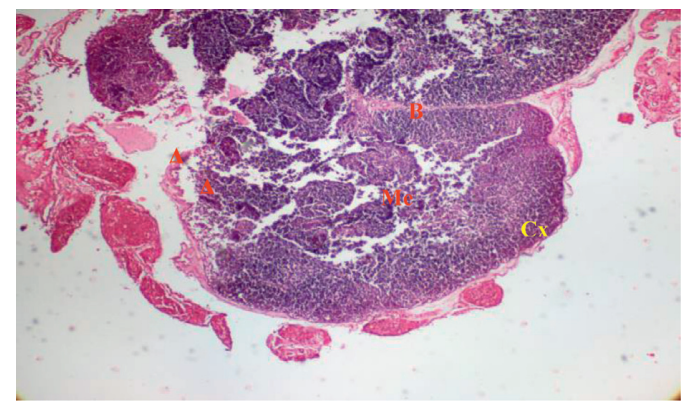

(a)

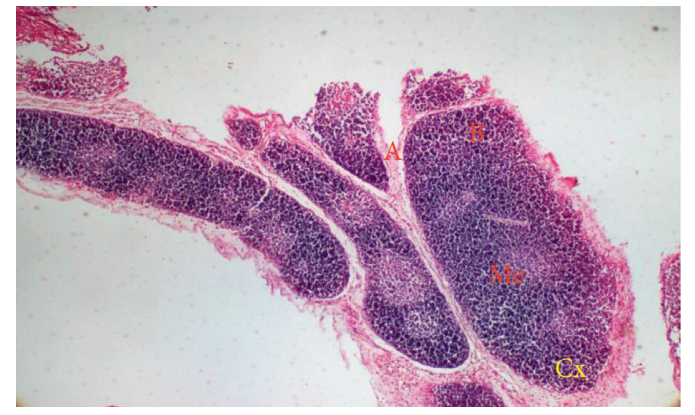

(c)

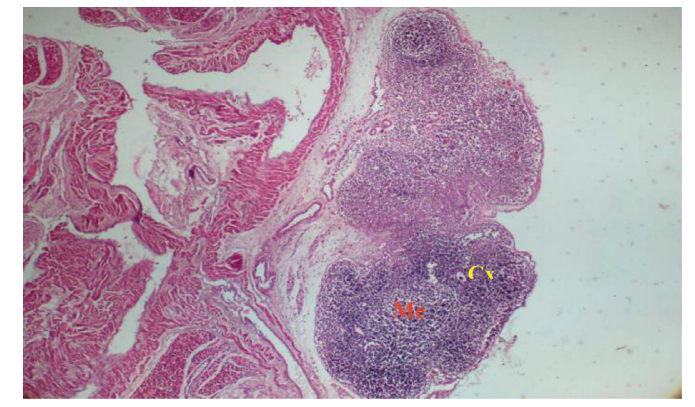

(b)

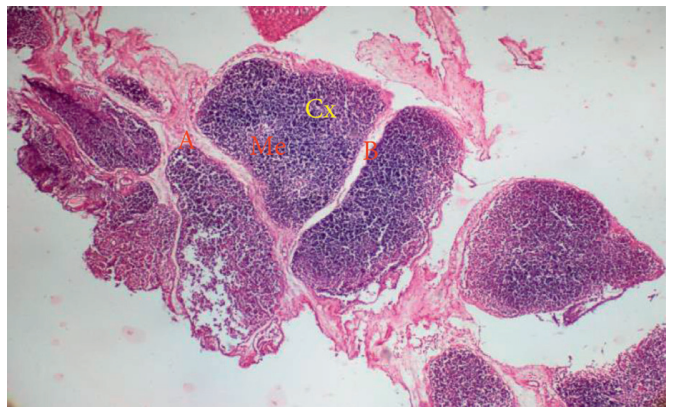

(d)

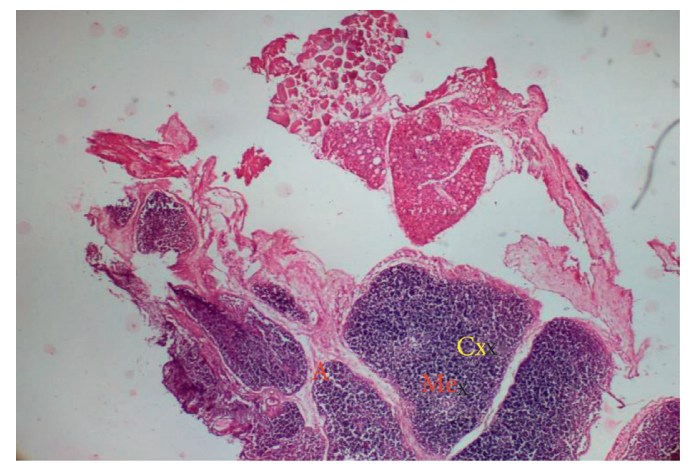

(e)

FiguRE 3: Microarchitecture of rat thymus of different groups (control and extract-treated groups). Normal group: untreated animals; negative: animals receiving methylprednisolone only. (a) Cross section in a rat thymus of normal group showing a lobule of the thymus with dense cortex $(\mathrm{Cx})$, medulla $(\mathrm{Me})$, interlobular $(\mathrm{A})$, and intralobular (B) (H\&E, X 100). (b) Cross section in a rat thymus of negative group showing the lobule of the thymus with less dense cortex $(\mathrm{Cx})$ and the medulla (Me), interlobular (A), and intralobular (B) (H\&E, X 100). (c) Cross section in a rat thymus of $25 \mathrm{mg} / \mathrm{kg}$-treated group showing the lobule of the thymus with dense cortex $(\mathrm{Cx})$ and the medulla $(\mathrm{Me})$, interlobular (A), and intralobular (B) (H\&E, X 100). (d) Cross section in a rat thymus of $50 \mathrm{mg} / \mathrm{kg}$-treated group showing the lobule of the thymus with dense cortex $(\mathrm{Cx})$ and the medulla (Me), interlobular (A), and intralobular (B) (H\&E, X 100). (e) Cross section in a rat thymus of $100 \mathrm{mg} / \mathrm{kg}$-treated group showing the lobule of the thymus with very dense cortex $(\mathrm{Cx})$ and the medulla (Me), interlobular (A), and intralobular (B). (H\&E, X 100). 


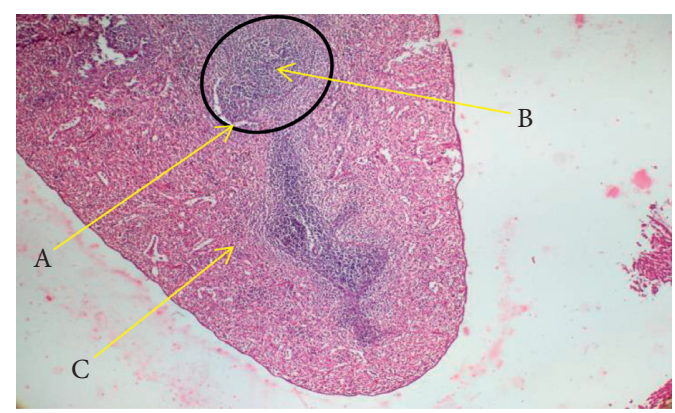

(a)

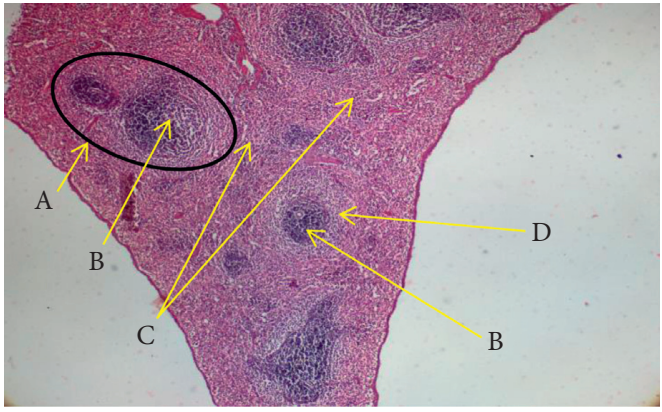

(c)

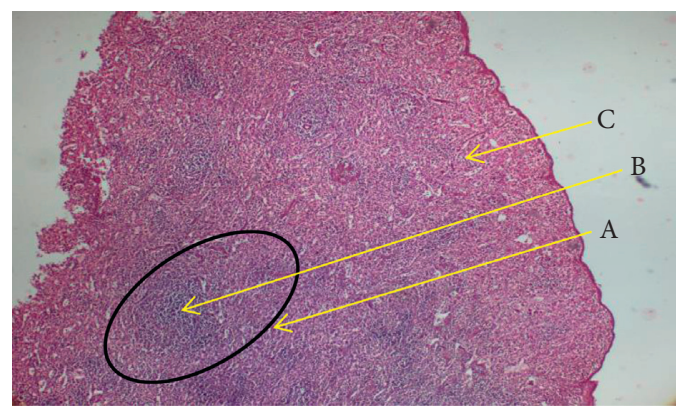

(b)

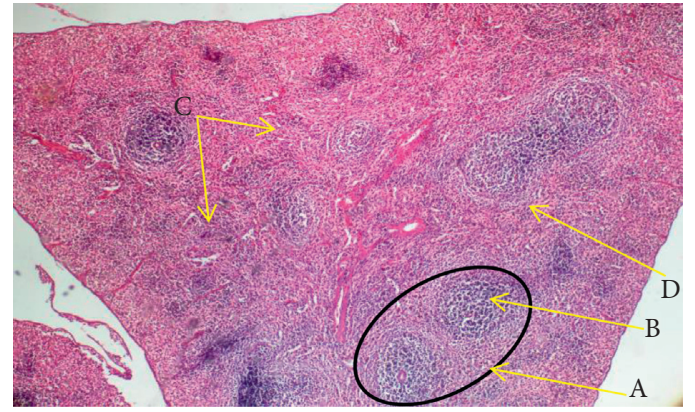

(d)

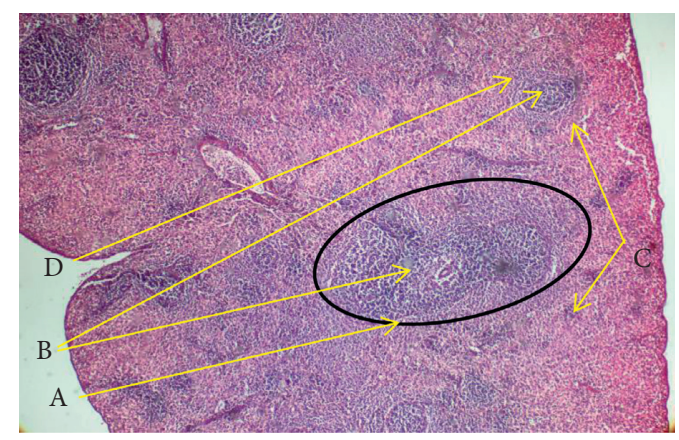

(e)

Figure 4: Microarchitecture of rat spleen of different groups (control and extract-treated groups). Normal group: untreated animals; negative: animals receiving methylprednisolone only. (a) Cross section of a rat spleen of control group showing the white pulp of the spleen with germinal center (B) and the red pulp (C) (H\&E, X 100). (b) Cross section of a rat spleen of negative group showing white pulp (A) with poor germinal center (B) and the red pulp (C) (H\&E, X 100). (c) Cross section of a rat spleen of $25 \mathrm{mg}$ group showing white pulp (A) with very dense germinal center (B), marginal zone (D), and the red pulp (C) (H\&E, X 100). (d) Cross section of a rat spleen of $50 \mathrm{mg}$ group showing white pulp (A) with dense germinal center (B), marginal zone (D), and the red pulp (C) (H\&E, X 100). (e) Cross section of a rat spleen of $50 \mathrm{mg}$ group showing white pulp (A) with dense germinal center (B), marginal zone (D), and the red pulp (C) (H\&E, X 100).

vitro [28], and macrophage metabolism has been also reported as a crucial indicator for the immune function [29]. This activity of macrophages is affected by the methylprednisolone [30, 31]. Thus, the results of this study indicate the potential immunostimulatory effect of $T$. le-testui, through which it may stimulate the immune cells and improve the body's immune function [32, 33]. Methylprednisolone is also known to cause lymphocytic depletion in spleen in rats [34]. The polysaccharide-rich extract of $T$. le-testui, furthermore, demonstrated that it can improve the thymus and spleen indices in rats treated with this immunosuppressive drug. This is evidence that T. le-testui improves the strength of the body's innate immune function [35-37]. This effect of T. le-testui is also supported by the lymphocytes colonization of thymus and spleen as demonstrated by the histological analysis of these organs reported for other mushrooms species such as Lentinula edodes [38]. As a result of phagocytes activation, reactive oxygen species and nitrogen reactive species are generally produced to participate in the intracellular killing of pathogens and mediate inflammatory reaction [39]. The results of this study also showed that polysaccharide-rich extract of T. le-testui has a potential to increase the NO and total radical compounds in methylprednisolone-treated rats. Once more, this demonstrated that polysaccharides of $T$. le-testui can boost the body's immune system and can reverse the symptoms of low immunity.

In conclusion, the polysaccharide-rich extract showed potent antioxidants and immunomodulatory effects on different components of the innate and adaptive immune response. These pharmacological activities can justify the 
traditional use of this mushroom for the treatments of several diseases in relation to the immune response.

\section{Data Availability}

Data will be available under request to the corresponding author.

\section{Conflicts of Interest}

The authors declare that they have no conflicts of interest.

\section{References}

[1] C. L. Hobbs, Medicinal Mushrooms: An Exploration of Tradition, Healing and Culture, Botanica Press, Santa Cruz, Calif, 1995.

[2] T. Christopher, O. Mahamat, M. N. Chungong, C. Atemenkeh Ngwa, and P. Maishu Samka, "Immunological activities of crude polysaccharides extracts of climacodon pulcherrimus (phanerochaetaceae) in lipopolysaccharide-induced rat macrophages and neutrophils' responses," Journal of $\mathrm{Me}$ dicinal Food, vol. 24, pp. 1-8, 2020.

[3] M. Oumar, AL. Njouonkou, C. Tume, A. M. Abamukong, and A. Kamanyi, "Assessment of antimicrobial and immunomodulatory activities of termite associated fungi, Termitomyces clypeatus R. Heim (Lyophyllaceae, Basidiomycota)," Jounal of Clinical Phytoscience, vol. 1, pp. 1-15, 2018.

[4] B.-Z. Zaidman, M. Yassin, J. Mahajna, and S. P. Wasser, "Medicinal mushroom modulators of molecular targets as cancer therapeutics," Applied Microbiology and Biotechnology, vol. 67, no. 4, pp. 453-468, 2005.

[5] N. W. Paul, M. G. Eliud, M. G. Benson, and M. O. Keziah, "Antimicrobial activity of mushroom (agaricus bisporus) and fungal (trametes gibbosa) extracts from mushrooms and fungi of egerton main campus, njoro Kenya," Journal of Biomedical Sciences, vol. 6, no. 3, pp. 1-6, 2017.

[6] W. Xu, F. Zhang, Y. Luo, L. Ma, X. Kou, and K. Huang, "Antioxidant activity of a water-soluble polysaccharide purified from Pteridium aquilinum," Carbohydrate Research, vol. 344, no. 2, pp. 217-222, 2009.

[7] A. Shanmugam, T. S. Mahalakshm, and A. Barwin Vi, "Antimicrobial activity of polysaccharide isolated from the cuttlebone of sepia aculeata (orbingy, 1848) and sepia brevimana (steenstrup, 1875): an approach to selected antimicrobial activity for human pathogenic microorganisms," Journal of Fisheries and Aquatic Science, vol. 3, no. 5, pp. 268-274, 2008.

[8] J. Shen, M. Tanida, Y. Fujisaki, Y. Horii, K. Hashimoto, and K. Nagai, "Effect of the culture extract of Lentinus edodes mycelia on splenic sympathetic activity and cancer cell proliferation," Autonomic Neuroscience, vol. 145, no. 1-2, pp. 50-54, 2009.

[9] T. A. Wynn, A. Chawla, and J. W. Pollard, "Macrophage biology in development, homeostasis and disease," Nature, vol. 496, no. 7446, pp. 445-455, 2013.

[10] X. Huiwen, C. Xueru, F. Yijun, and L. Aoxue, "Antioxidant activity of water-soluble polysaccharides from brasenia schreberi," Pharmacognosy Magazine, vol. 12, no. 47, pp. 193-197, 2016.

[11] Y. M. Liu, Z. M. Qi, S. Y. Wang, and S. X. Liu, "An overview on natural polysaccharides with antioxidant properties," Current Medicinal Chemistry, vol. 20, no. 23, 2013.
[12] Z. Quanbin, Y. Pengzhan, L. Zhien, Z. Hong, X. Zuhong, and L. Pengcheng, "Antioxidant activities of sulfated polysaccharide fractions from Porphyra haitanesis," Journal of Applied Phycology, vol. 15, pp. 305-310, 2003.

[13] G. A. Yoboue, L. S. Kouassi, J. Suomion, T. B. Sea, and K. C. Karim, "Nutritional potential of the edible wild mushroom termitomyces letestui from bouafle (Cote d'Ivoire)," World Journal of Advance Health Research, vol. 4, no. 3, pp. 31-35, 2020.

[14] M. Oumar, C. Tume, A.-L. Njouonkou, J. Amadoh, N. Nfor, and A. Kamanyi, "Screening of the immunomodulatory and antibacterial activity of Termitomyces letestui (Pat.) Heim (Lyophyllaceae), an edible mushroom from Cameroon," J Basic Clin Physiol Pharmacol, vol. 29, no. 6, pp. 645-650, 2018.

[15] M. Yuhan, H. Huaqi, W. Jingzhu, W. Chunyang, C. Kuanglin, and H. Qing, "Assessment of polysaccharides from mycelia of genus ganodermaby mid-infrared and near-infrared spectroscopy," Scientific Reports, vol. 8, p. 10, 2018.

[16] T. Masuko, A. Minami, N. Iwasaki, T. Majima, S.-I. Nishimura, and Y. C. Lee, "Carbohydrate analysis by a phenol-sulfuric acid method in microplate format," Analytical Biochemistry, vol. 339, no. 1, pp. 69-72, 2005.

[17] M. Z. Bhatti, A. Ali, A. Ahmad, S. Asma, A. Saeed, and S. A. Malik, "Antioxidant and phytochemical analysis of Ranunculus arvensis L. extracts," BMC Research Notes, vol. 8, no. 1, pp. 279-287, 2015.

[18] H. Kato, I. E. Lee, N. Van Chuyen, S. B. Kim, and F. Hayase, "Inhibition of nitrosamine formation by nondialyzable melanoidins," Agricultural \& Biological Chemistry, vol. 51, no. 5, pp. 1333-1338, 1987.

[19] R. J. Ruch, S.-J. Cheng, and J. E. Klaunig, "Prevention of cytotoxicity and inhibition of intercellular communication by antioxidant catechins isolated from Chinese green tea," Carcinogenesis, vol. 10, no. 6, pp. 1003-1008, 1989.

[20] H. Kikuzaki and N. Nakatani, "Antioxidant effects of some ginger constituents," Journal of Food Science, vol. 58, no. 6, pp. 1407-1410, 1993.

[21] P. Honghui, H. Yuanyuan, H. Jiguo et al., "Purification and identification of a polysaccharide from medicinal mushroom Amauroderma root with immunomodulatory activity and inhibitory effect on tumor growth," Impact Journal, vol. 6, no. 19, pp. 1-8, 2015.

[22] S. Pinaki, B. Piyasha, A. Lakshmisona et al., "Simple modified colorimetric methods for assay of total oxidative stress and antioxidant defense in plasma: study in diabetic patients," iMedPub Journals, vol. 7, no. 5, pp. 1-7, 2015.

[23] N. Sayed, M. R. Khan, and M. Shabbir, "Antioxidant activity, total phenolic total and flavonoid contents of whole plant extract Torilis leptophylla L." BMC Complementary and Alternative Medicine, vol. 12, p. 112, 2012.

[24] P. Pacher, J. S. Beckman, and L. Liaudet, "Nitric oxide and peroxynitrite in health and disease," Physiological Reviews, vol. 87, no. 1, pp. 315-424, 2007.

[25] S. Yadav, M. Bhargav, R. L. Ramya, and H. Nowfal, "In-Vitro antioxidant and immunomodulatory activity of Citrullus lanatus seed," International Journal of Engineering Sciences \& Research Technology, vol. 5, no. 12, pp. 679-685, 2016.

[26] B. Taha, H. Selma, B. Houda, B. Insaf, S. Noomene, and L. Ahmed, "Alleviated actions of plantago albicans extract on lead acetate-produced hepatic damage in rats through antioxidant and free radical scavenging capacities," Journal of Medicinal Food, vol. 00, no. 0, pp. 1-15, 2020. 
[27] H. Xiao, X. Cai, Y. Fan, and A. Luo, "Antioxidant activity of water-soluble polysaccharides from brasenia schreberi," Pharmacognosy Magazine, vol. 12, no. 47, pp. 193-197, 2016.

[28] H. Wei, Y. Xu, B. Cheng, and Y. Xiong, "Synergistic effects of Lactobacillus rhamnosus ZDY114 and bovine colostrums on the immunological function of mouse in vivo and in vitro," Applied Microbiology and Biotechnology, vol. 75, no. 2, pp. 427-434, 2007.

[29] O. Mahamat, H. Flora, C. Tume, and A. Kamanyi, "Immunomodulatory activity of momordica charantia L. (Cucurbitaceae) leaf diethyl ether and methanol extracts on Salmonella typhi-infected mice and LPS-induced phagocytic activities of macrophages and neutrophils," Evidence-based Complementary and Alternative Medicine: eCAM, vol. 2020, Article ID 5248346, 11 pages, 2020.

[30] W. W. Chatham and R. P. Kimberly, "Treatment of lupus with corticosteroids,” Lupus, vol. 10, no. 3, pp. 140-147, 2001.

[31] J. J. Carolyn, J. M. Karen, and I. V. Malcolm, "Prednisolone inhibits phagocytosis by polymorphonuclear leucocytes via steroid receptor mediated events," Annals of the Rheumatic Diseases, vol. 42, pp. 56-62, 1983.

[32] X. Zhou, Q. Dong, X. Kan et al., "Immunomodulatory activity of a novel polysaccharide from Lonicera japonica in immunosuppressed mice induced by cyclophosphamide," PLoS One, vol. 13, no. 10, p. e0204152, 2018.

[33] Q. Bao, L. Qian, C. Gong, and X. Shen, "Immune-enhancing activity of polysaccharides from gastrodiaelata," Journal of Food Processing and Preservation, vol. 41, p. 13016, 2017.

[34] S.-X. Yan, X.-M. Deng, Q.-T. Wang, X.-J. Sun, and W. Wei, "Prednisone treatment inhibits the differentiation of B lymphocytes into plasma cells in MRL/MpSlac-lpr mice," Acta Pharmacologica Sinica, vol. 36, no. 11, pp. 1367-1376, 2015.

[35] L. Shuling, W. Litao, R. Qiang et al., "Immunomodulatory and antioxidant activities of a polysaccharide from ligustrum vicaryi L. Fruit," Evidence-based Complementary and Alternative Medicine, vol. 2020, p. 10, 2020.

[36] L. Barrea, C. Di Somma, G. Muscogiuri et al., "Nutrition inflammation and liver-spleen axis, critical review," Journal of Food Science Nutrition, vol. 58, no. 18, pp. 3141-3158, 2018.

[37] F. P. Baqai, D. S. Gridley, J. M. Slater et al., "Effects of spaceflight on innate immune function and antioxidant gene expression," Journal of Applied Physiology, vol. 106, no. 6, pp. 1935-1942, 2009.

[38] L. Chen, "Co-inhibitory molecules of the B7-CD28 family in the control of T-cell immunity," Nature Reviews Immunology, vol. 4, no. 5, pp. 336-347, 2004.

[39] F. N. Razali, A. Ismail, N. Z. Abidin, and A. S. Shuib, "Stimulatory effects of polysaccharide fraction from Solanum nigrum on RAW 264.7 murine macrophage cells," PLoS One, vol. 9, no. 10, p. e108988, 2014. 